Saudi Journal of Medicine

Abbreviated Key Title: Saudi J Med ISSN 2518-3389 (Print) |ISSN 2518-3397 (Online)

\title{
Biochemical Markers Affecting Left Ventricular Ejection Fraction in Patients Younger Than 35 Years of Age with Established Coronary Artery Disease
}

\author{
Shwan Othman Amen, MD, $\mathrm{PhD}^{1}$, Banan Qasim Rasool ${ }^{2 *}$ \\ ${ }^{1}$ Interventional Cardiology, Consultant Interventional Cardiologist, Surgical Specialty Hospital - Cardiac Center, Erbil, Iraq \\ ${ }^{2}$ M.B.Ch.B, Junior House Officer, Erbil Teaching Hospital, Hawler Medical University, Erbil, Iraq
}

DOI: $\underline{10.36348 / \mathrm{sjm} .2020 . \mathrm{v} 05 \mathrm{i} 12.005}$

| Received: 01.12.2020 | Accepted: 09.12.2020 | Published: 18.12.2020

*Corresponding Author: Dr. Banan Qasim Rasool

\section{Abstract}

Background: Cardiac functioning and assessment in patients with established CAD can be done through performing Echocardiography and calculating the Ejection Fraction, which is an important and vital tool in clinical practice and related to prognosis. Left Ventricular Ejection Fraction (LVEF) can predict future clinical outcome and assist in risk stratification. Objective: The aim of this research was to evaluate the relationship between lower EF with clinical and biochemical markers, and to determine the predictors of left ventricular EF in patients with established CAD whose younger than 35 years' age. Patients and Methods: This study was enrolled in Surgical Specialty Hospital- Cardiac Center / Erbil-Iraq among 100 consecutive patients (77 males and 23 females) between November 2016 to December 2017 of those whose age was $\leq 35$ years old. Results: The mean age was 31.6 \pm 2.91 years with an Age range of 24-35 years. 22.9\% of those with Anterior Wall Myocardial Infarction (AWMI) and 22.7\% of those with Inferior Wall Myocardial Infarction (IWMI) had left ventricular Ejection fraction (LVEF) of less than 44\% $(\mathrm{P}=0.0001) .33 .3 \%$ of Patients with STEMI on presentation, later had LVEF of $\leq 44 \%(P=0.0001)$. Lower LVEF $(\leq 44 \%)$ was observed more in male gender and smoker patients with established CAD $(P<0.05)$. 29\% of those with 10-fold S. Troponin level elevation on presentation, had LVEF $\leq 44 \%$ and $45.2 \%$ of them had LVEF of between $45 \%-54 \%(P=0.001) .37 .5 \%$ of those with Very High LDL levels had EF $\leq 44 \%(P=0.02)$. After performing Coronary Angiography, $68.8 \%$ of those with poor functioning of heart (LVEF $\leq 44 \%)$ had LAD lesion. $(P<0.05)$. Conclusion: We concluded that there was evidence of a strong relationship (Inversely Proportional) between Poor EF with high LDL and Markedly Elevated Serum Troponin of more than 10 folds, young Patients with AWMI and IWMI had Poor functioning of the heart. (LVEF of $\leq 44)$.

Keywords: Left Ventricular Ejection Fraction, Myocardial Infarction, prognostic Factors after MI, Serum Troponin and Poor EF, LDL and Poor EF.

Copyright (C) 2020 The Author(s): This is an open-access article distributed under the terms of the Creative Commons Attribution 4.0 International License (CC BY-NC 4.0) which permits unrestricted use, distribution, and reproduction in any medium for non-commercial use provided the original author and source are credited.

\section{Abbreviation List}

$\mathrm{CAD}=$ Coronary Artery Disease

LVEF = Left Ventricular Ejection Fraction

$\mathrm{AMI}=$ Acute Myocardial Infarction

AWMI = Anterior Wall Myocardial Infarction

IWMI = Inferior Wall Myocardial Infarction

LAD = Left Anterior Descending

RCA $=$ Right Coronary Artery

STEMI $=$ ST Elevation Myocardial Infarction

NSTEMI $=$ Non ST Elevation Myocardial

Infarction

\section{INTRODUCTION}

Coronary Artery Disease (including STEMI, NSTEMI and Unstable Angina) is a major non communicable disease and a major cause of death worldwide with an estimated annual incidence rate of seven million people [1]. The coronary vessels damage can be caused by an array of crucial risk factors such as hypertension, dyslipidemia, diabetes mellitus and smoking cigarettes $[2,3]$.

As a result of coronary artery occlusion, CAD usually leads to death if complicated by severe heart failure, malignant ventricular arrhythmia, or cardiac rupture [4]. Despite the application of percutaneous coronary intervention (PCI) and conventional medicine, $\mathrm{CAD}$ patients remain at certain risk of in-hospital death and complications as well as recurrent acute cardiovascular events [4-6]. Epidemiological studies have revealed men to be more affected by CAD compared to women. Moreover, female patients are believed to have more symptom burden and a higher 
rate of functional disability but a lower prevalence of CAD. In patients with coronary artery disease, men tend to have a higher lipid core compared with women [7].

Previous studies were done to investigate serum total cholesterol in relation to left ventricular ejection fraction and CAD where they found out higher total cholesterol and higher HDL are associated with higher left ventricular ejection fraction $[8,9]$.

Left ventricular ejection fraction (LVEF) is the simplest and most widespread parameter to measure left ventricular (LV) systolic dysfunction and the main driver for categorizing heart failure-as a consequence of Myocardial Infarction. Although LVEF has many acknowledged limitations, it is still pivotal for the management, risk stratification and follow-up of patients with cardiovascular diseases, being easy to obtain and non-invasive [10]. Still, there are some pathophysiological arguments for an underrating of LVEF as a reliable index of systolic function [11]. Moreover, the degree of the impairment in LV systolic function provides information about the extent of the damage directed to the myocardium, while it is not a clue to the underlying cause [12].

Assessment of LV systolic function is bound to remain a key element in patient prognostication in the next years. With this respect, a refinement in the evaluation of LV systolic function could be achieved by using global longitudinal strain [13, 14], overcoming some limitations linked to LVEF measurements like geometric assumptions and technical issues, such as apical foreshortening and difficulties in proper delineation of the endocardial borders. Nonetheless, to further reduce morbidity and mortality of patients with $\mathrm{CAD}$, it seems likely that we need to introduce into clinical practice some novel markers, possibly linked to therapeutic optimization either in the peri-procedural and in the chronic setting.

In our study, we used a prospective clinical observational design to find and analyze the association between Left Ventricular Ejection Fraction (LVEF) and LDL and Serum Troponin-I, to determine the predictors of LVEF.

\section{MATERIALS AND METHODS Study Population}

The study population comprised 100 consecutive patients with different presentations of Coronary Artery Disease (STEMI, NTEMI and Unstable Angina) admitted to the causality department of the Surgical Specialty Hospital - Cardiac Center within $12 \mathrm{~h}$ of the onset of clinical signs and symptoms from Nov 2016 to Dec 2017. All patients aged $\leq 35$ years were included in this study. Diagnostic criteria of CAD were followed according to the guidelines of the European Society of Cardiology, such as when the patient is presented with typical chest pain, diagnostic electrocardiogram (ECG) changes, and a significant elevation of cardiac enzymes. Exclusion criteria for patients and controls included pregnancy, anemia, and kidney failure. Patients subsequently underwent coronary angiography and revascularization by either primary PCI or pharmaco-invasive PCI or CABG surgery after obtaining informed consent with Their Family.

\section{Statistical Analysis}

The statistical analysis was done using IBM SPSS Statistics, Version 23.0. Armonk, NY: IBM Corp, USA. Baseline patient characteristics were summarized. All data are presented as mean \pm SD for continuous variables and $\mathrm{n}(\%)$ for categorical variables. Comparisons between groups were made using Pearson correlation or Spearman's rho for continuous variables and Fisher exact test for categorical variables. A Pvalue of $<0.05$ was considered statistically significant. A multivariate linear regression model was done to determine the predictors of LVEF.

\section{Ethical Consideration}

All the data has been anonymous and not the relevant personal information's of the patients has been shown. The data was not collected from the patients directly, but it was collected from the Admission File of the patients (including a copy of all the investigations performed) and consent was taken from the Data Registry Department of SSH-Cardiac Center.

The patient's personal states, name and other direct indicators have been anonymousized earlier.

\section{Laboratory Analysis}

The serum levels of lipid profile parameters, including total cholesterol (TC), HDL-C, LDL-C, and TG, were determined at the admission of the patient presented with AMI. These laboratory tests were carried out using Cobas c311 chemistry analyzer (Roche Diagnostics, Basel, Switzerland) for sample analysis. Hyperlipidemia was defined as serum cholesterol of $\geq$ $200 \mathrm{mg} / \mathrm{dl}$, triglyceride $(\mathrm{TG})>200 \mathrm{mg} / \mathrm{dl}$, low-density lipoprotein $>130 \mathrm{mg} / \mathrm{dl}$, HDL-C $<50 \mathrm{mg} / \mathrm{dl}$ for female and $<40 \mathrm{mg} / \mathrm{dl}$ for male, for known cases of dyslipidemia and/or those on medication for dyslipidemia.

Elevated levels of serum Troponin was calculated of more than $0.015 \mathrm{ng} / \mathrm{dl}$ and CK-MB of > $6.2 \mathrm{ng} / \mathrm{dl}$ according to the SSH-Cardiac Center laboratory normal ranges.

\section{Echocardiography}

All index transthoracic echocardiographs were recorded during routine clinical practice according to the current guidelines. Two-dimensional M-mode echocardiography was performed within 3 days of the PCI. In each patient, LVEF was measured using bidimensional echocardiography from two- and four- 
chamber apical views by the modified Simpson's method. The wall motion score in-ex was derived according to a 17 segment model

\section{Coronary Angiogram}

Coronary angiograms were performed using standard techniques. Significant coronary artery disease (CAD) was defined as $\geq 70 \%$ stenosis of an epicardial coronary artery. The extent of CAD was characterized by one-, two-, or three-vessel disease or left main disease. Percutaneous coronary intervention (PCI) was performed according to the physician's discretion.
Patients Demographic and Clinical Characteristic

In this current study, 100 consecutive patients with different manifestation of CAD (STEMI, NSTEMI and Unstable Angina) of which $77 \%$ male and $23 \%$ female, were enrolled. The mean age was $31.6 \pm 2.9$ years, with an age range of 24-35-year-old. The mean $\pm \mathrm{SD}$ was $31.2 \pm 2.9$ years in male, and $32.9 \pm 2.4$ year in female. The prevalence of $\mathrm{CAD}$ and its major manifestation-AMI was higher in male patients comparing to female patients with a statistically significant difference $(P<0.05)$. This indicates that younger males experience $\mathrm{CAD}$ and its manifestations more than females. The data summarized in Table-1.

\section{RESULTS}

Table-1: Shows the prevalence of Coronary Artery Diseases in relation to Age Groups and Gender

\begin{tabular}{|l|l|l|l|l|l|l|}
\hline \multirow{2}{*}{$\begin{array}{l}\text { Age groups } \\
\text { (Year) }\end{array}$} & \multicolumn{2}{|l|}{ Male } & \multicolumn{2}{l|}{ Female } & \multicolumn{2}{l|}{ Total } \\
\cline { 2 - 7 } & No. & \% & No. & \% & No. & \% \\
\hline$\leq 24$ & 3 & 3.9 & 0 & 0 & 3 & 3 \\
\hline $25-29$ & 16 & 20.8 & 2 & 8.7 & 18 & 18 \\
\hline$\geq 30$ & 58 & 75.3 & 21 & 91.3 & 79 & 79 \\
\hline Total & 77 & 100 & 23 & 100 & 100 & 100 \\
\hline
\end{tabular}

The highest prevalence rate of CAD was seen in patients within age group of 30-35 years (79\%), followed by $18 \%$ in age group of $25-29$ years then a smaller portion of $3 \%$ among those $\leq 24$ year of age.

It was observed that the majority of the male patients $75.3 \%$ with CAD belong to the age group of 30-35 years. On comparison, $91.3 \%$ of female patients belong to the age group of $30-35$ years. $(P=0.2)$

The mean Left Ventricular Ejection Fraction was $53.5 \pm 8.85$. The prevalence of poor LVEF $(\leq 44 \%)$ among male patients was $20.8 \%$ while $33.8 \%$ had Fair LVEF (45\%-54\%) and 45.5\% had Good LVEF ( $\geq 55 \%$ ). On the other hand, $26.1 \%$ of Female patients had Fair LVEF (45\%-54\%) and 73.9\% had Good LVEF ( $\geq 55 \%)$ showing a statistically significant difference $(P=0.024)$.

With analyzing the data, the results showed a clear and obvious Relationship between Left Ventricular Ejection Fraction with LDL and Serum Troponin- I in patients younger $\leq 35$ years with any form of established CAD. We hypothesized that the factors influencing left ventricular ejection fraction for patients with coronary artery disease to be LDL-C, Serum Troponin-I. We conducted correlation tests using Spearman's rho to assess the relation- ship between LVEF, LDL-C, Serum Troponin-I in patients with CAD.

Low density lipoprotein-c (LDL-c) had significant inverse relationship with LVEF ( $\mathrm{r}=-0.27$, $\mathrm{P}=0.029)$ and Serum Troponin-I $(\mathrm{r}=-0.415, \mathrm{P}=0.001)$.
Explaining more about the relationship of Poor LVEF with Lipid parameters and Cardiac markers (mainly Serum Troponin-I and CK-MB), results showed that among the patients with Poor LVEF $(\leq 44 \%), 29 \%$ had 10 folds' increase in serum Troponin-I $(P=0.001)$, $17.5 \%$ had Elevated levels of CK-MB $(P=0.12), 37.5 \%$ had very high $\operatorname{LDL}(P=0.029), 30.8 \%$ had reduced HDL $(P=0.2), 26.7 \%$ had High total cholesterol $(P=$ $0.1)$ and $25 \%$ had high levels of Triglyceride $(P=0.3)$.

Interestingly, the prevalence of Risk factors of CAD in patients with Poor LVEF $(\leq 44 \%)$ showed $26.5 \%$ were smoker $(P$ 0.02), 36\% were hypertensive $(P<0.05)$ and $22.7 \%$ were Diabetic $(P=0.4)$. It was observed that $22.9 \%$ of AWMI and $22.7 \%$ of IWMI patients later had LVEF of $\leq 44 \%$ and this showed a statistically significant difference. $(P=0.001)$. Young patients who presented with STEMI had a prevalence of $33.3 \%$ Poor LVEF and only $4 \%$ of patients who presented with NSTEMI had Poor LVEF. $(P<0.05)$

Focusing of the angiographic findings, among the young patients $(\leq 35$ years of age) who had Poor LVEF $(\leq 44 \%), 68.8 \%$ of them had occlusion in LAD artery, $12.5 \%$ had occlusion in LCX artery and only $6.3 \%$ had occlusion in RCA. And this was strongly significant statistically showing $P$ value of $<0.05$.

Table-2 summarizes the relation of Poor LVEF with other variables and showing their statistical significance's ( $P$ value) 
Table-2: Shows the Relation between Poor Left Ventricular Ejection Fraction (LVEF) $\leq 44 \%$ With Other Variables and Their $P$ Value

\begin{tabular}{|l|l|l|}
\hline Variables & Prevalence in \% & $\boldsymbol{P}$ value \\
\hline AWMI & 22.9 & 0.001 \\
\hline IWMI & 22.7 & $<0.05$ \\
\hline Elevated Troponin & 29 & 0.001 \\
\hline Elevated CK-MB & 17.5 & 0.12 \\
\hline High LDL & 37.5 & 0.029 \\
\hline Reduced HDL & 30.8 & 0.2 \\
\hline High Total Cholesterol & 26.7 & 0.1 \\
\hline High Triglyceride & 25 & 0.3 \\
\hline STEMI & 33.3 & $<0.05$ \\
\hline NSTEMI & 4 & $<0.05$ \\
\hline Smoking & 26.5 & 0.02 \\
\hline Hypertension & 36 & 0.005 \\
\hline DM & 22.7 & 0.4 \\
\hline LAD occlusion & 30.6 & 0.001 \\
\hline RCA occlusion & 11.1 & 0.001 \\
\hline LCX occlusion & 66.7 & 0.001 \\
\hline
\end{tabular}

\section{DISCUSSION}

The current research indicated a strong inversely proportional relationship between Left Ventricular Ejection Fraction (LVEF) with LDL-c, and Troponin-I in patients with CAD. To the best our knowledge, this is the first research in our region, to reveal the relationship between LVEF and biomarkers. Previous studies have shown that hyperlipidemia adversely affected the LVEF, particularly in patients with CAD. They showed an important relationship between LVEF and HDL, suggesting that HDL might influence left ventricular systolic function through further-atherosclerotic mechanisms because they observed that LVEF was adversely influenced by dyslipidemia irrespective of the severity of coronary atherosclerosis [15-18]. In our study, which involved patients younger than 35 years of age with coronary artery disease, we found out LDL to be inversely proportional with LVEF and it was statistically significant. Meaning one-unit change increase in LDL is associated with a unit decrease in LVEF percentage. In other words, the higher the LDL level, the lower the LVEF. Another study showe similar results about the inverse relationship of LVEF and levels of LDL [26]. This shows our study concur with previous findings in other researches.

Furthermore, one of the Cardiac biomarkers, Troponin-I which is a power indicator of myocardial necrosis, has been studied before and was found to be inversely proportional with LVEF in patients with CAD. They found out that the LVEF of $<50 \%$ was predicted by troponin I concentration of $>6.6 \mathrm{ng} / \mathrm{ml}$. In another research analysis, it showed that patients with LVEF $<35 \%$ had the highest level of troponin I and vice versa. They also showed that the LVEF had a negative correlation with troponin I levels $(\mathrm{r}=-0.54, \mathrm{P}$ $=0.001)$ [19-21].
Results of another study showed that there was a significant negative relationship between troponin I and LVEF in patients with CAD [22]. Our results about the relationship between LVEF and Cardiac markers were similar to the mentions researches above, showing significant strong relation between them, in which any one-unit change increase in Serum Troponin I levels was associated with a unit decrease in LVEF percentage.

Moreover, there were studies done in the area of determination of predictors of LVEF. Whereby, their analysis demonstrated that several traditional and easily available factors were associated with a greater risk of heart failure development, even among low-risk CAD population. Some of the studies showed lipids predicted the severity of new onset CAD in type-2 DM patients and not in relation to LVEF. In another research, low HDL-c was strongly predictive of cardiovascular events in patients with coronary artery disease [23-25].

In this current study we found out that patients with $\mathrm{LVEF} \leq 44 \%$ had prevalence of cardiovascular risk factors as; $36 \%$ were hypertensive, $26.5 \%$ were smoker and $22.7 \%$ were DM

We believe by sharing these findings of our study, will empower the clinicians with knowledge on CAD, by exploring the relationship between LVEF and biomarkers, and predictors of LVEF in these patients. Moreover, the degree of the impairment in LV systolic function, provides information about the extent of the damage directed to the myocardium, while it is not a clue to the underlying cause [12].

\section{CONCLUSION}

There was a strong inversely proportional relationship between LVEF with Lipid profile, mainly LDL and Cardiac Markers Mainly Serum Troponin I. 


\section{Authors Contribution}

Drafting of manuscript and data analysis was done by: BQ Rasool. Critical revision and correction were done by: SO Amen. All authors read and approved the final manuscript.

\section{ACKNOWLEDGMENT}

We would like to thank all the people who played an important role in accomplishing this work. We would like to appreciate the staff of SSH-Cardiac Centre who were supportive in collecting the data.

\section{Study Limitations}

Our study had some limitations within which some of the consecutive patients had to be excluded from the population because of some missing data.

Secondly, the data was all from a single center and not all the patients from local centers involved.

Lastly but not least, results of this study may not be completely generalizable because the sample size was restricted to a time period because of availability of the data.

Disclosure: The authors have no conflicts of interest to disclose

Founding: This Article has not been founded by any organization/Institution and has no Financial Support.

\section{REFERENCES}

1. White, H. D., \& Chew, D. P. (2008). Acute myocardial infarction. The Lancet, 372(9638), 570-584.

2. Gander, J., Sui, X., Hazlett, L. J., Cai, B., Hébert, J. R., \& Blair, S. N. (2014). Peer Reviewed: Factors Related to Coronary Heart Disease Risk Among Men: Validation of the Framingham Risk Score. Preventing chronic disease, 11:E 140.

3. Zhang, Y., Liu, Y., Zhang, H., \& Zhou, J. (2019). Impact of sex-specific differences in calculating the pretest probability of obstructive coronary artery disease in symptomatic patients: a coronary computed tomographic angiography study. Coronary artery disease, 30(2), 124-130.

4. Libby, P. (2001). Current concepts of the pathogenesis of the acute coronary syndromes. Circulation, 104(3), 365-372.

5. Ma, X. J., Yin, H. J., \& Chen, K. J. (2009). Appraisal of the prognosis in patients with acute myocardial infarction treated with primary percutaneous coronary intervention. Chinese journal of integrative medicine, 15(3), 236-240.

6. Chen, K. J., Hui, K. K., Lee, M. S., \& Xu, H. (2012). The potential benefit of complementary/alternative medicine in cardiovascular diseases. Evid Based Complement Alternat Med. 2012:125029.
7. Löffler, A. I., \& Bourque, J. M. (2016). Coronary microvascular dysfunction, microvascular angina, and management. Current cardiology reports, $18(1), 1$.

8. Mayala, H. A., Bakari, K. H., Mghanga, F. P., \& ZhaoHui, W. (2018). Clinical significance of PETCT coronary flow reserve in diagnosis of nonobstructive coronary artery disease. BMC research notes, 11(1), 566.

9. Wang, T. D., Wu, C. C., Chen, W. J., Lee, C. M., Chen, M. F., Liau, C. S., \& Lee, Y. T. (1998). Dyslipidemias have a detrimental effect on left ventricular systolic function in patients with a first acute myocardial infarction. The American journal of cardiology, 81(5), 531-537.

10. Passino, C., \& Vergaro, G. (2019). Left ventricular ejection fraction and coronary artery disease in the era of precision medicine. European Journal of Preventive Cardiology. 26(12):1271-1272.

11. Konstam, M. A., \& Abboud, F. M. (2017). Ejection fraction: misunderstood and overrated (changing the paradigm in categorizing heart failure). Circulation, 135(8), 717-719.

12. Packer, M. (2016). The room where it happens: a skeptic's analysis of the new heart failure guidelines. Journal of Cardiac Failure, 22(9), 726-730.

13. Guerra, F., Stronati, G., Fischietti, C., Ferrarini, A., Zuliani, L., Pomponio, G., ... \& Gabrielli, A. (2018). Global longitudinal strain measured by speckle tracking identifies subclinical heart involvement in patients with systemic sclerosis. European journal of preventive cardiology, 25(15), 1598-1606.

14. Soufi Taleb Bendiab, N., Meziane-Tani, A., Ouabdesselam, S., Methia, N., Latreche, S., Henaoui, L., ... \& Benkhedda, S. (2017). Factors associated with global longitudinal strain decline in hypertensive patients with normal left ventricular ejection fraction. European Journal of Preventive Cardiology, 24(14), 1463-1472.

15. Mayala, H. A., Bakari, K. H., Mghanga, F. P., \& ZhaoHui, W. (2018). Clinical significance of PETCT coronary flow reserve in diagnosis of nonobstructive coronary artery disease. BMC research notes, 11(1), 566.

16. Wang, T. D., Wu, C. C., Chen, W. J., Lee, C. M., Chen, M. F., Liau, C. S., \& Lee, Y. T. (1998). Dyslipidemias have a detrimental effect on left ventricular systolic function in patients with a first acute myocardial infarction. The American journal of cardiology, 81(5), 531-537.

17. Kempen, H. J., Van Gent, C. M., Buytenhek, R., \& Buis, B. (1987). Association of cholesterol concentrations in low-density lipoprotein, highdensity lipoprotein, and high-density lipoprotein subfractions, and of apolipoproteins AI and AII, with coronary stenosis and left ventricular function. The Journal of laboratory and clinical medicine, 109(1), 13-18. 
18. Wang, T. D., Lee, C. M., Wu, C. C., Lee, T. M., Chen, W. J., Chen, M. F., ... \& Lee, Y. T. (1999). The effects of dyslipidemia on left ventricular systolic function in patients with stable angina pectoris. Atherosclerosis, 146(1), 117-124.

19. Somani, D., Gahlot, R. S., Lakhotia, M., Choudhary, C. R., \& Sangavi, S. (2005). Troponin I measurement after myocardial infarction and its correlation with left ventricular ejection fraction: a prospective study. JIACM. 6(1):38-41.

20. Khan, M. H., Islam, M. N., Aditya, G. P., Islam, M. Z., Bhuiyan, A. S., Saha, B., ... \& Rahman, R. (2017). Correlation of Troponin-I Level with Left Ventricular Ejection Fraction and In-hospital outcomes after First Attack of Non-ST Segment Elevation Myocardial Infarction. Mymensingh Medical Journal: MMJ, 26(4), 721-731.

21. Rao, A. C. R., Collinson, P. O., Canepa-Anson, R., \& Joseph, S. P. (1998). Troponin T measurement after myocardial infarction can identify left ventricular ejection of less than $40 \%$. Heart, $80(3)$, 223-225.

22. Mayala, H. A., Mafuru, M., Mkangala, A., Mayala, M., Pallangyo, P., Minja, D., ... \& Zhaohui, W. (2020). Factors influencing left ventricular ejection fraction in patients with coronary microvascular disease and obstructive coronary artery disease. BMC Research Notes, 13(1), 1-6.

23. Lewis, E. F., Solomon, S. D., Jablonski, K. A., Rice, M. M., Clemenza, F., Hsia, J., ... \& Gersh, B. J. (2009). Predictors of heart failure in patients with stable coronary artery disease: a PEACE study. Circulation: Heart Failure, 2(3), 209-216.

24. Du, Y., Chen, J., Chen, M. H., Yang, S. H., Li, S., Guo, Y. L., ... \& Li, J. J. (2016). Relationship of lipid and lipoprotein ratios with coronary severity in patients with new on-set coronary artery disease complicated with type 2 diabetics. Journal of geriatric cardiology: JGC, 13(8), 685-692.

25. Miller, M., Seidler, A., Kwiterovich, P. O., \& Pearson, T. A. (1992). Long-term predictors of subsequent cardiovascular events with coronary artery disease and'desirable'levels of plasma total cholesterol. Circulation, 86(4), 1165-1170.

26. Chen, Y., He, X. M., Meng, H., Zhao, Q. Z., Zhen, Y. Z., Tian, L., ... \& Liu, G. (2014). Relationship between lipids levels and right ventricular volume overload in congestive heart failure. Journal of geriatric cardiology: JGC, 11(3), 192-199. 